\title{
Sexual dimorphism of the pelvic architecture: A struggling response to destructive and parsimonious forces by natural \& mate selection
}

\author{
Aaron Leong*
}

\section{INTRODUCTION}

Sexual dimorphism of the human pelvis is linked intimately with its adaptive functions. The peculiarly shaped hominid pelvis represents the total response to the diverse forces that have moulded its structure. These diverse forces are requirements for efficient bipedalism and parturition. In some respects, the structural demands of these unrelated functions have been in conflict. The morphological response to the dominant requirement, bipedalism, is clearly discernible, while the changes serving the needs of parturition are seen as compensatory modifications as reflected with greater emphasis for pelvic sexual dimorphism in the female. In addition, sexual selection has made sexual dimorphism even more pronounced. The female buttocks have undergone sexual elaboration through mate choice by males. Thus, total pelvic architecture is a mosaic constituted of the aggregate of differential responses to different functional goals.

There are complications during parturition that have been repeatedly prevented or interceded by medical technology, close monitoring, surgical practices such as caesarian sections, and other strategies. With these complications, one could hypothesize that these natural operators, which have exerted their influence since the beginning of mankind, might be becoming increasingly destabilized, attenuated or stochastic.

\section{The Male and Female Pelvic Blueprint and Anatomic Variations \\ In general, the structure of the male pelvis is}

* To correspondence should be addressed: Aaron Leong Si Arn BSc (Physiology) 2002, MD CM 2007, McGill University, 1537

Summerhill Ave, Apt \#208, Montreal Quebec H3H 1C2

E-mail Address: aaron.leong@mail.mcgill.ca significantly heavier and thicker than that of the female. The male pelvic bones are also adapted to fit a more massive and sturdy body architecture. For example, the male acetabulum has been designed to fit a bigger femur. Though a large amount of the sexual dimorphism of the pelvis is accounted for by size differences, sexlinked shape variation is also very conspicuous and cannot be considered an allometric consequence of differences in body size between the sexes (1). These variations in shape are demonstrated by the more rounded frame of the female pelvis. The sciatic notches are broader, the greater pelvis is shallower, the lesser pelvis is wider and the pelvic inlet and outlet are larger (longer pubic bones and a greater degree of curvature of the pectineal line). The female hipbones are also different in traits associated in the position of the sacroiliac joint in the iliac bone (2). As a result of this 'flattened' appearance, the female obturator foramen is more elliptical. The pubic arch is formed by the conjoined rami of the pubis and ischium of the two sides. These rami meet at the pubic symphysis to form the subpubic angle. The subpubic angle is nearly a right angle in females and is considerably less in males; approximately $30^{\circ}$ narrower (3).

Superficial to the skeleton and musculature of the pelvis, sexual dimorphism in pelvic morphology is most apparent in body fat distribution as measured by waist hip ratio (WHR). The WHR has been shown to be independent of overall body weight and an accurate predictor of risk for various diseases, premature mortality, degree of estrogenicity and fecundity of women (4). Undoubtedly, healthy women have a greater propensity to possess rounder hips and a lower WHR compared to most men (4).

Nonetheless, the human pelvis is not always 
distinctly dimorphic. It has been well established that nature has allowed individual anatomical variation and departures from set norms within each sex. Hence, one could infer that it is possible to find any of the previously defined archetypal features in the 'wrong' sex. In addition, there is metric and morphologic variation in the expression of sexual dimorphism between racial phenotypes and populations (5). In other words, the final shape of the female pelvis is affected by multiple etiological factors - cultural, environmental and genetic.

The gynecoid pelvis (rounded shape) is said to be the normal female type while the android pelvis (heartshaped) is often designated a male variant. Women with the android pelvis do not typically present with hyperandrogenism, and signs of hyperandrogenism have been similarly encountered in patients with gynecoid and other types of pelvis. This android pelvis was also found more frequently in women exposed to strenuous physical activity during adolescence and observed more often when the acquisition of erect posture was delayed beyond the usual age of 14 months, while a platypelloid pelvis was more frequent when erect posture was acquired before 14 months (6).

In addition to being developmentally discriminating, the distribution of the anthropoid pelvis between both sexes and frequency is ethnically discriminating as well. It was observed that there were significant differences in the accuracy of sex determination from pelvic morphology between both males and females, and whites and blacks. Pubic bone shape was the easiest to assess and was the most consistently reliable morphological indicator of sex in both sexes and population groups. However, in blacks, the greater sciatic notch form allowed the highest separation. This demonstrates that racial differences significantly affect the expression of sexual dimorphism (5).

\section{Temporal Development of Sexual Dimorphism of the Pelvis}

Sexual dimorphism begins as early as the fetal stage of development. Indeed, the appearance of morphological differences, such as the greater interischiatic distance in fetal females after the 26-27th week of gestation, could indicate the presence of pathological fetal development (7). Evidently, sexual dimorphism does become increasingly pronounced and divergent as a child grows into an adult. While there are significant sex differences in breadth of the ischium and acetabular regions by 8 years of age, most of the sexual dimorphism in the pelvis develops during the adolescent growth spurt, during which both male and female pelves undergo growth remodeling of the pelvic cavity. Over the same time period, males show significantly greater growth in the acetabulum, and females show greater growth in the pelvic cavity (8).

By age 18, the pelvis demonstrates a posterior-toanterior gradient of increasing dimorphism within the inlet of the pelvic birth canal. Canalization of growth of the transverse diameters of the sacrum, inlet, anterior inferior iliac spines, and breadths of the ilium and ischium during puberty can be attributed to the effects of stabilizing selection operating on both males and females. On the other hand, over time, there is evidence of increased variation and discordant change within each sex and differential growth between the sexes for the interacetabular diameter, breadths of the anterior superior and posterior inferior iliac spines, public length, and ilium height. These are patterns indicating effects of disruptive selection on the pelvis (8). Growth studies have indicated that the linea terminalis may be unique by continuing to grow in early adulthood in females but not in males. This growth occurs at the medial border of the pubis. The selective advantage of a later age at maturation of the pubis in females than males is that the period of growth is prolonged, thereby contributing to sexual dimorphism in pubic length, linea terminalis length and pelvic inlet circumference (9).

\section{Natural Adaptive Forces in Shaping Sexual Dimorphism of the Pelvis: \\ Bipedalism versus Parturition}

An evolutionary pattern towards bipedalism, taking into account selective pressures of reproduction, has been demonstrated by the increase of critical dimensions of the pelvis as the maternal skeleton becomes larger. One such dimension, the distance between the ischial spines, defines the pelvic midplane and is an important consideration in hominid reproduction. It was found that a correlation exists between skeletal frame size and the distance between the ischial spines in females. In females, but not in males, weight and femoral head diameter are excellent predictors of the distance between ischial spines. However, the femoral head diameter, in females, does not predict weight as well as it does in males (10).

These adaptations to ease parturition as reflected in the sexual dimorphism in the human pelvis and femur are disadvantageous to women in terms of mechanics of locomotion. The mechanical variables that primarily contribute to dimorphism are the moment arm of the gluteus medius and the torque produced by the abductors at the hip. These mechanical aspects of hip function produce greater pressure on the femoral head in females (11).

The divergent selective forces shaping the pelvis remain destabilized primarily because of the rare and capricious growth of the human brain. The pelvis has to 
resculpt itself repeatedly to deliver babies with a disproportionately large head. Perpetual brain expansion is a consequence of both natural and cultural evolution that favours intelligence, learning, tool making and an elaborate culture. Naturally, it is easy to see that the speed and uniqueness of this encephalization process must have been driven by some sort of positive feedback mechanism. One of the postulated processes is the runaway social competition model ${ }^{1}$ which implies that our brain developed as a result of social competition between fellow men. Humans got better at deceiving and outmaneuvering one another which, as a result, selected for men who were able to see through deceptive plans. Such a 'runaway' process would result in a rapid positive feedback mechanism that enhances human brain power. Another 'runaway' model that could explain this encephalization is the runaway gene-culture coevolution model ${ }^{2}$. This model assumes that the emergence of modern culture that depended heavily on the manipulation of tools and materials meant that only individuals who were creative and capable enough to develop tools and harness or exploit materials survived. These individuals were naturally those with higher cognitive abilities and were thus able to further improve and complicate the material culture, which in turn selected for even brighter men (12).

As humans have evolved from a unique line of primate ancestry, which accentuates the need for brain expansion, innovation and intelligence in place of physical strength, agility, speed and endurance, the present-day fetal head is painfully massive and cumbersome for the mother during childbirth. This is substantiated by the classic comparative relation, which states that primate species, like humans, with a large neonatal brain size relative to the birth canal, tend to have more sexually dimorphic pelves (13).

\section{Mate Selection in Sculpting the Human Body:}

\section{How do the Waist-Hip-Ratio and Body Mass Index}

Signify Healthiness?

While the natural forces discussed above are quintessential operators in hominid evolution, the influence of mate selection on sexual dimorphism in the modern-day human are apparent and cannot be discounted. Body traits classically considered to be attractive, such as the lower Waist-Hip-Ratio (WHR) and Body Mass Index (BMI) of women, could be explained by mate selective operators.

While mate choice takes much time and energy, sometimes to the point of impairing survival or genetic perpetuation, it is very evolutionarily sound. A number of us have probably contemplated delaying procreation, and running the risk of never being able to propagate, simply because the ideal mate has not crossed our paths. Why are we willing to suffer such risks? One possible response to this existential question is that if we were to find an ideal mate with good genes, our offspring would likely have higher survivability, and propagate its genes well. According to the "selfish gene" hypothesis, animals with genes to select mates with good genes would hence produce more viable offspring carrying those selective genes, and thus the genes for selectivity would spread throughout the gene pool.

Evolutionary biology theories, such as the good genes model $^{3}$, have suggested that the most fundamental form of mate choice is selection for indicators of viability and fertility, which may manifest in any easily perceivable bodily or behavioural trait to reveal age, health, nutritional status, strength, dominance, social status and disease resistance. These honest indicators would demonstrate the chances that a potential mate has desirable genetic traits that would be passed onto offspring and enhance their survival, or is capable of helping to provide for and protect offspring. Some of these indicators that serve as major targets for selective mate choice by males include facial neoteny, averageness and symmetry. Another functional model of mate selection, the good provider model ${ }^{4}$, pertains to the more fluid and complex social patterns of hominid civilization. Thus, indicators of social success and cognition would also come into play as survival depends not only on individual strengths but also on the ability to cooperate with and outmaneuver others (14).

These mate selection theories could explain the reason behind the morphological amplification of the

1 The runaway social competition model suggests that rapid encephalization occurred so that hominids could predict and manipulate each other's behavior, leading to a social intelligence arms race between mind reading and deception. Runaway models are hypothetical and are difficult to prove.

2 The runaway gene-culture co-evolution model suggests that hominids got smarter to learn and use material culture. As the material culture got more complex, hominids had to evolve to be even more intelligent with each generation.

3 The Good Genes Model proposes that choice criteria are functional and desirable characteristics are evidence of health and well-being. Human beings would evolve to recognize only honest indicators as true representations of fitness since those that were capable of doing so would have mated with the truly fit individuals and produced more offspring.

4 The good-provider model states that characteristics are selected based on the functional benefits to the bearer of the child. This might have led to the female strategy of finding a nice, dependable, stable long-term male and the male strategy of finding a female with engorged breasts and a nurturing personality to feed and take care of young. It also suggests that females would be choosier than males because of their higher parental investment during pregnancy. Even so, this model is merely theoretical and does not necessarily reflect the realities of hominid society. 
female breasts and buttocks. In order for females to solicit male attention and investment, accentuated bodily features signaling youthfulness, healthiness and fertility would have to be judged as 'attractive' to the opposite sex. Indeed, this is a fundamental assumption of adaptive explanations of female attractiveness. In particular, sexual dimorphism in body fat distribution has also been assumed to be vital in mate selection. WHR is an accurate predictor of nutritional status, reproductive age and degree of estrogenicity and parity of women, independent of overall body weight. Finally, cross-cultural and historical data have suggested that the relationship between WHR and female attractiveness is not culture-specific and not inculcated by what modern Western fashion dictates or media (15).

The other putative cue to female physical attractiveness is BMI. It has been shown that both males and females assigned higher ranking for attractiveness, youthfulness, healthiness, reproductive capability and intelligence to normal weight figures with low WHRs. Overweight figures were assigned low rankings for all these qualities except reproductive capability. Underweight figures, regardless of WHR, were assigned low ranking for reproductive capability and those underweight figures that had high WHRs were assigned low ranking for healthiness $(16,17)$. Female and male subjects, judged heavier female target figures with low WHRs as more attractive and healthier than thinner figures with higher WHRs. Female subjects perceived heavier female target figures with low WHR to represent ideal female figures. It is proposed that female attractiveness and ideal female shape may be more influenced by WHR than overall body size (4).

More recently, it was revealed in another study that WHR was less important than BMI as a predictor of attractiveness ratings for bodies. Viewers' judgments were influenced more by BMI than WHR (18). BMI was the primary predictor of attractiveness in both front and profile, and the putative visual cues to BMI showed a higher degree of view-invariance than shape cues (19). Interestingly, a study in 2004 developed the volume height index (VHI), which is the body volume divided by the square of the height. This was heralded as the most important and direct visual determinant of female physical attractiveness. VHI is also a key indicator of health and fertility owing to its strong linear relation to $\mathrm{BMI}(20)$.

\section{COMPLICATIONS DURING CHILDBIRTH:}

\section{Are Female Hips too Small?}

While, the influence of natural forces and mate selection on human anatomy have been well documented for years, few have explored the possibility that rapid advancement of medical practices might currently play an important role in increasing the frequency of more atypical anatomical forms and consequently, re-shape many of our human characteristics, such as the female pelvic anatomy.

It has been previously established that the size and shape of the pelvic inlet are key during labour as it determines the ease in which the fetal head enters the lesser pelvis. Along these lines, the size of the lesser pelvis has always been important in obstetrics because it determines the size of the bony pelvic canal through which the fetus passes during a vaginal delivery (3). More frequently than one might imagine, some kind of difficulty or injury, across a spectrum of severity and recovery rates, is encountered during human childbirth. Today, a woman with a narrow or misshaped pelvis can, in fact, successfully give birth to a massive baby. Such a phenomenon would probably never have existed before the age of aggressive medical and scientific intervention in assisting the birthing process. In fact, for our ancestors, a grossly mismatched maternal-fetal presentation was often sorrowfully fatal to both mother and child.

Even with modern surgical and medical practices, childbirth, a rite of passage for most women, is dangerous and can potentially lead to lifelong morbidity. During parturition, extensive damage of varying severity can occur to the pelvic floor, which supports the fetal head while the cervix of the uterus is dilating. The fetal head may compress the nerves of the mother's sacral plexus, producing pain in the lower limbs. Tearing or stretching of the perineal body during childbirth may result in a permanent weakness of the pelvic diaphragm. The perineal body is especially important in women because it is the final support of the pelvic viscera and serves as the attachment for the perineal muscles. As a result, prolapse of the vagina through the vaginal orifice may occur after the support of the inferior part of the posterior wall of the vagina is removed (3).

These ill-effects associated with childbirth are also exacerbated when the mother faces obstructed labor. The major causes of obstructed labor is cephalopelvic disproportion, which may be due to a small pelvis, a large baby, fetal malpresentation, a tight perineum, or abnormalities or tumors of the uterus, ovary, or vagina. When obstructed labor is unrelieved, the presenting fetal part is impacted against the soft tissues of the pelvis and a widespread ischemic vascular injury develops that result in tissue necrosis and subsequent fistula formation. (21). The processes of labour and vaginal delivery, especially the former, can cause pudendal nerve damage. A heavier baby and a longer second stage of labour were both associated with 
significant prolongation of pudendal nerve latency. Pudendal nerve damage was also found after forceps delivery and perineal tears (22).

Vaginal delivery has also been regarded as one of the commonest causes of stress urinary incontinence and anal incontinence. This is primarily caused by injury to the levator ani, in particular the pubococcygeus, which encircles and supports the urethra, the vagina and anal canal, and the pelvic fascia, which may be torn during childbirth. Stress urinary incontinence is further provoked by damage to the levator ani and pelvic fascia that hold the position of the neck of the bladder and the urethra in place. Furthermore, delivery causes partial denervation of the pelvic floor, with consequent renervation, in most women having their first baby. For some, it is likely to be the first step along a path leading to prolapse or severe stress urinary incontinence $(3,22,23)$. Anal incontinence has additionally been attributed to traumatic childbirths and multiple deliveries, because of repeated stretching of the pudendal nerves and subsequent nerve damage (24). The risk for anal sphincter injury alone could be used as an argument for elective Cesarean section (25).

In light of the plethora of injuries associated with childbirth, it is easy for one to postulate that in the absence of healthcare, incompatible pelvic-fetal anatomy could result in significant infant and maternal mortality and morbidity. Although concrete scientific evidence does not exist, one has to wonder if medical practice is indirectly and insidiously introducing variability to the human anatomy.

\section{CONCLUSION}

The morphology of the human body is susceptible to perpetual modifications. This dynamic nature is characteristic of most biological systems and testimony to traditional theories of evolution. However, classic Darwinism never fully accounted for or addressed the potency of the overbearing forces put forth by mate selection and social survival strategies. Intelligence, which evolved as a natural survival strategy, heralds a new tangential path in evolution. Henceforth, modern concepts of biocultural evolution are ironically replacing Darwinism to address and explain present-day issues. Old strategies of symbiosis, competition for ecological niches, survival of the fittest, phenotypic variability and mutations have lost some of their pertinence in the dawn of human civilization which seems to cherish altruism, cooperation, supra-ordinate goals and social cohesiveness. It is also prudent for us to acknowledge that even as we strive to analyze attractiveness and attempt to find functional patterns or algorithms of beauty, clichés like 'beauty is in the eye of its beholder' and 'beauty is not only skin deep' are timeless and accurate.

\section{REFERENCES}

1. Jordaan HV. The differential development of the hominid pelvis. S Afr Med J 1976; 50(19): 744-748.

2. Arsuaga JL, Carretero JM. Multivariate analysis of the sexual dimorphism of the hip bone in a modern human population and in early hominids. Am J Phys Anthropol. 1994; 93(2): 241-257.

3. Moore KL, Dalley AF. Clinically oriented anatomy (4th Ed). Lippincott Williams \& Wilkins; 1999.

4. Singh D. Adaptive significance of female physical attractiveness: role of waist-to-hip ratio. J Pers Soc Psychol 1993; 65(2): 293-307.

5. Patriquin ML, Loth SR, Steyn M. Sexually dimorphic pelvic morphology in South African whites and blacks. Homo 2003; 53(3): 255-262.

6. Abitbol MM. The shapes of the female pelvis. Contributing factors. J Reprod Med 1996; 41(4): 242-250.

7. Merrot T, Panuel M, Bourliere B, Kathia C, Philip N, Dutour O. Expression of sexual dimorphism in the fetal pelvic girdle. $\mathrm{C} \mathrm{R}$ Acad Sci III 2001; 324(2): 137-141.

8. LaVelle M. Natural selection and developmental sexual variation in the human pelvis. Am J Phys Anthropol 1995; 98(1): 59-72.

9. Tague RG. Maternal mortality or prolonged growth: age at death and pelvic size in three prehistoric Amerindian populations. Am J Phys Anthropol 1994; 95(1): 27-40.

10. Walrath DE, Glantz MM. Sexual dimorphism in the pelvic midplane and its relationship to Neandertal reproductive patterns. Am J Phys Anthropol 1996; 100(1): 89-100.

11. Burr DB, Van Gerven DP, Gustav BL. Sexual dimorphism and mechanics of the human hip: a multivariate assessment. Am J Phys Anthropol 1977; 47(2): 273-278.

12. Miller GF. The Mating Mind: How Sexual Choice Shaped the Evolution of Human Nature, Heinemann; 2000.

13. Ridley M. Brief communication: pelvic sexual dimorphism and relative neonatal brain size really are related. Am J Phys Anthropol 1995; 97(2): 197-200.

14. Gangestad, S. W., Thornhill, R. The Evolutionary Psychology of Extrapair Sex; the Role of Fluctuating Asymmetry. Evolution and Human Behavior 1997; 18: 69-88.

15. Singh D. Female Mate Value at a Glance: Relationship of Waistto-Hip Ratio to Health, Fecundity and Attractiveness. Neuroendocrinol Lett. 23 Suppl 2002; 4: 81-91.

16. Singh D. Waist-to-hip ratio and judgment of attractiveness and healthiness of female figures by male and female physicians. Int J Obes Relat Metab Disord 1994; 18(11): 731-737.

17. Singh D. Ideal female body shape: role of body weight and waist-to-hip ratio. Int J Eat Disord 1994; 16(3): 283-288.

18. Tovee MJ, Hancock PJ, Mahmoodi S, Singleton BR, Cornelissen PL. Human female attractiveness: waveform analysis of body shape. Proc R Soc Lond B Biol Sci. 7 2002; 269(1506): 2205-2213.

19. Tovee MJ, Cornelissen PL. Female and male perceptions of female physical attractiveness in front-view and profile. Br J Psychol. 2001; 92(Part 2): 391-402.

20. Fan J, Liu F, Wu J, Dai W. Visual perception of female physical attractiveness. Proc R Soc Lond B Biol Sci. 22;271(1537):347$52 ; 2004$

21. Arrowsmith S, Hamlin EC, Wall LL. Obstructed labor injury complex: obstetric fistula formation and the multifaceted morbidity of maternal birth trauma in the developing world. Obstet Gynecol Surv. 1996; 51(9): 568-574.

22. Sultan AH, Kamm MA, Hudson CN. Pudendal nerve damage during labour: prospective study before and after childbirth. $\mathrm{Br}$ 
J Obstet Gynaecol. 1994; 101(1): 22-28.

23. Morley R, Cumming J, Weller R. Morphology and neuropathology of the pelvic floor in patients with stress incontinence. Int Urogynecol J Pelvic Floor Dysfunct 1996; 7(1): 3-12.

24. Willis S, Faridi A, Schelzig S, Hoelzl F, Kasperk R, Rath W, Schumpelick V. Childbirth and incontinence: a prospective study on anal sphincter morphology and function before and early after vaginal delivery. Langenbecks Arch Surg. 2002; 387(2): 101-107.

25. Faridi A, Willis S, Schelzig P, Siggelkow W, Schumpelick V, Rath W. Anal sphincter injury during vaginal delivery--an argument for cesarean section on request? J Perinat Med. 2002; 30(5): 379-387.

Aaron Leong is a third year medical student at McGill University Faculty of Medicine. He graduated with a B.Sc. in Physiology from the National University of Singapore in 2002. His research interests include sociobiology and medical education. 